\title{
Diagnósticos de enfermagem de pacientes com necessidade de locomoção afetada internados em uma unidade hospitalar
}

\author{
Nursing diagnoses of hospital inpatients with impaired transfer ability
}

Diagnósticos de enfermería en pacientes con necesidad de locomoción afectada internados en unidad hospitalaria

Maria Joanna D'arc de Melo França ${ }^{1}$, Suzana de Oliveira Mangueira², Jaqueline Galdino Albuquerque Perrelli ${ }^{3}$, Simara Lopes Cruz ${ }^{4}$, Marcos Venícios de Oliveira Lopes ${ }^{5}$

\footnotetext{
${ }^{1}$ Enfermeira. Vitória de Santo Antão, PE, Brasil. E-mail: melojoanna3@hotmail.com.

${ }^{2}$ Enfermeira, Mestre em Enfermagem. Discente do Programa de Pós-Graduação em Enfermagem, nível Doutorado, da Universidade Federal do Ceará (UFC). Professora Assistente da Universidade Federal do Pernambuco (UFPE). Vitória de Santo Antão, PE, Brasil. E-mail: suzanaom@hotmail.com.

${ }^{3}$ Enfermeira, Mestre em Enfermagem. Discente do Programa de Pós-Graduação em Neuropsiquiatria, nível Doutorado, UFC. Professora Assistente da

UFPE. Vitória de Santo Antão, PE, Brasil. E-mail:jaquelinealbuquerque@hotmail.com.

${ }^{4}$ Fonoaudióloga, Mestre em Saúde Pública. Professora Assistente da UFPE. Vitória de Santo Antão, PE, Brasil. E-mail: simara.cruz@hotmail.com.

${ }^{5}$ Enfermeiro, Doutor em Enfermagem. Professor Associado da UFPE. Fortaleza, CE, Brasil. E-mail: marcos@ufc.br.
}

\section{RESUMO}

O estudo teve como objetivos identificar os diagnósticos de enfermagem mais frequentes e classificar o nível de independência em atividades da vida diária de pacientes com locomoção afetada internados em uma unidade de clínica médica. Trata-se de um estudo descritivo com abordagem quantitativa que utilizou dois instrumentos estruturados para coleta de dados aplicados a 63 pacientes. Os dados coletados foram examinados com base no processo de raciocínio diagnóstico proposto por Gordon e, posteriormente, foram formulados os diagnósticos com base na Taxonomia da NANDA-I. Foram identificados 44 diagnósticos. Estiveram presentes em todos os pacientes os diagnósticos: deambulação prejudicada, mobilidade física prejudicada, risco de infecção e risco de quedas. O estudo mostrou que pacientes com locomoção afetada apresentam número elevado de diagnósticos de enfermagem e alto nível de dependência em atividades da vida diária.

Descritores: Enfermagem; Diagnóstico de Enfermagem; Locomoção.

\section{ABSTRACT}

The objective of this study was to identify the most frequent nursing diagnoses and classify the level of independence for performing activities of daily living in patients with impaired transfer ability, hospitalized in a medical clinic. This descriptive study was performed with quantitative approach, and used two structured instruments to collect data from 63 patients. The collected data were examined based on the diagnosis rationale proposed by Gordon and, later, diagnoses were formulated based on NANDA-I Taxonomy. A total 44 diagnoses were identified. The following diagnoses were present in all patients: impaired walking, impaired physical mobility, risk for infection and risk for falls. The study showed that patients with impaired transfer had a high number of nursing diagnoses and a high dependency level in activities of daily living.

Descriptors: Nursing; Nursing Diagnosis; Locomotion.

\section{RESUMEN}

El estudio objetivó identificar los diagnósticos de enfermería más frecuentes y clasificar el nivel de independencia en actividades de la vida diaria de pacientes con locomoción física afectada, internados en unidad de clínica médica. Estudio descriptivo con abordaje cualitativo, que utilizó dos instrumentos estructurados para colectar los datos, aplicados a 63 pacientes. Los datos recogidos fueron examinados en base al proceso de razonamiento diagnóstico propuesto por Gordon y, posteriormente, fueron formulados los diagnósticos de acuerdo a la Taxonomía de la NANDA-I. Fueron identificados 44 diagnósticos. Estuvieron presentes en todos los pacientes los diagnósticos: Deterioro de la deambulación, Deterioro de la movilidad física, Riesgo de infección y Riesgo de caídas. El estudio mostró que los pacientes con la motricidad afectada presentan un número elevado de diagnósticos de enfermería y alto nivel de dependencia en actividades de la vida diaria.

Descriptores: Enfermería; Diagnóstico de Enfermería; Locomoción. 


\section{INTRODUÇÃO}

A limitação física pode manifestar-se de forma súbita ou lentamente, conforme sua extensão e duração. É considerada um fator contribuinte para desencadear uma série de problemas de saúde, desde o déficit do autocuidado à interação social prejudicada, pode ainda agravar o curso clínico da doença de base e comprometer órgãos e sistemas corporais ${ }^{(1-2)}$. As principais complicações da imobilidade são alterações nos sistemas cardiovascular, respiratório, digestório, geniturinário, tegumentar e musculoesquelético ${ }^{(3)}$.

É responsabilidade da Enfermagem monitorar as funções fisiológicas para prevenir e detectar precocemente possíveis alterações. Para isso, utiliza-se o processo de enfermagem, que deve ocorrer em todos os âmbitos, individual e/ou coletivo, ambulatorial e/ou hospitalar, no ensino e na pesquisa ${ }^{(4)}$, regulamentado pela Resolução n 358/2009 do Conselho Federal de Enfermagem ${ }^{(5)}$

O processo de enfermagem reflete uma abordagem que permite o conhecimento mais completo e detalhado da situação que envolve a pessoa, suas reais necessidades e condições de enfrentamento dos problemas. Sua utilização deve ser norteada por uma teoria que guie a coleta de dados, facilite a identificação dos diagnósticos e embase as intervenções de enfermagem(6).

Na literatura atual são encontradas pesquisas que abordam diagnósticos de enfermagem em pacientes com necessidade de locomoção afetada em Unidade de Terapia Intensiva (UTI)e em idosos, principalmente ${ }^{(7-11)}$. Entretanto, existe uma lacuna no que se refere a estudos sobre diagnósticos de enfermagem em pacientes com esta necessidade internados em unidade de clínica médica.

Frente à importância que o tema assume para o trabalho do enfermeiro e ao considerar o grande número de pacientes com necessidade de locomoção afetada e o risco de desenvolverem problemas fisiológicos e psicossociais, bem como o elevado grau de dependência destes, desenvolveu-se este estudo que teve por objetivos identificar os diagnósticos de enfermagem mais frequentes e classificar o nível de independência em atividades da vida diária de pacientes com necessidade de locomoção afetada, internados em uma unidade de clínica médica.

\section{MÉTODO}

Trata-se de um estudo descritivo, com abordagem quantitativa, realizado em um hospital geral público. A população do estudo foi composta por pacientes adultos com necessidade de locomoção afetada, internados na unidade de clínica médica, de ambos os sexos, no período de agosto a dezembro de 2012.

Durante o período de coleta de dados, foram hospitalizados 297 pacientes na unidade de clínica médica. Destes, 111 apresentavam necessidade de locomoção afetada, identificados pelo pesquisador por meio da anamnese e avaliação física. Foram excluídos os pacientes com desorientação no tempo e no espaço. Aceitaram participar da pesquisa 63 pacientes, os quais compuseram a amostra do estudo.

Para a coleta de dados, foram utilizados dois instrumentos estruturados e validados. O primeiro instrumento teve a finalidade de coletar dados de enfermagem para indivíduos adultos hospitalizados em clínica médica ${ }^{(12)}$ e o segundo instrumento é a Escala de Independência em Atividades da Vida Diária (EIAVD) ou Escala de $\mathrm{Katz}^{(13)}$. O instrumento de coleta de dados para indivíduos adultos caracteriza os pacientes de acordo com suas necessidades/autocuidado, fatores de risco, exame físico e outras informações ${ }^{(12)}$. Já o segundo instrumento é uma adaptação transcultural da EIAVD para a língua portuguesa, no qual os itens apresentaram consistência interna e taxas confiáveis, o que comprovou ser equivalente à versão em inglês. A concordância corrigida para o acaso foi de 0,91 e o alfa de Chronbach variou de 0,80 a 0,92 . Esse instrumento foi desenvolvido para a avaliação dos resultados de tratamento em idosos e consta de seis itens que medem o desempenho do indivíduo nas atividades de autocuidado de acordo com uma hierarquia de complexidade, são eles: alimentação, controle de esfíncteres, transferência, higiene pessoal, capacidade para vestir-se e tomar banho(13).

Os instrumentos foram preenchidos por meio de anamnese e exame físico. Os dados coletados foram examinados com base no processo de raciocínio diagnóstico proposto por Gordon, que consiste nas seguintes etapas: coleta de informações, interpretação das informações, agrupamento de informações e denominação do agrupamento(14). Nesta última etapa, foram formulados os diagnósticos de enfermagem com base na taxonomia da NANDA-(15). O processo de 
raciocínio diagnóstico foi realizado de modo independente por dois pesquisadores. O primeiro pesquisador foi o mesmo que coletou e preencheu os instrumentos junto aos pacientes. $O$ segundo pesquisador, especialista em diagnóstico de enfermagem, realizou o processo de raciocínio diagnóstico a partir dos instrumentos preenchidos. Na sequência, foram comparados os diagnósticos de enfermagem encontrados e, quando houve divergência, os dois pesquisadores discutiram o caso até a obtenção do consenso. Os dados foram organizados em uma planilha do Microsoft Excel versão 2010, analisados por meio de estatísticas descritivas, apresentados em tabelas e discutidos com base na literatura.

Foram observados os princípios éticos durante a realização do estudo, dispostos na Resolução 196/96 do
Conselho Nacional de Saúde, o qual foi submetido à apreciação pelo Comitê de Ética em Pesquisa da instituição vinculada ao estudo e obteve aprovação com CAAE: 06324812.1 .0000 .5208 e $n^{\circ}$ do parecer: 140.904/2012. Os participantes foram esclarecidos quanto ao estudo e assinaram o Termo de Consentimento Livre e Esclarecido.

\section{RESULTADOS}

Conforme pode ser observado na Tabela 1, dos 63 pacientes em estudo, 36 (57,1\%) pertenciam ao sexo masculino. A maioria tinha entre 61 e 80 anos (média= 72,51 anos, desvio padrão=17,36 anos), era analfabeta, viúva, aposentada/pensionista e católica.

Tabela 1: Caracterização da amostra segundo dados socioeconômicos. Vitória de Santo Antão, PE, 2012.

\begin{tabular}{|c|c|c|}
\hline Variáveis & $\mathbf{N}^{\circ}$ & $\%$ \\
\hline \multicolumn{3}{|l|}{ Faixa etária (anos) } \\
\hline $21-40$ & 5 & 7,9 \\
\hline $41-60$ & 9 & 14,3 \\
\hline $61-80$ & 27 & 42,9 \\
\hline$>81$ & 22 & 34,9 \\
\hline \multicolumn{3}{|l|}{ Sexo } \\
\hline Masculino & 36 & 57,1 \\
\hline Feminino & 27 & 42,9 \\
\hline \multicolumn{3}{|l|}{ Escolaridade } \\
\hline Analfabeto & 28 & 44,4 \\
\hline Ensino fundamental incompleto & 27 & 42,9 \\
\hline Ensino fundamental completo & 1 & 1,6 \\
\hline Ensino médio incompleto & 1 & 1,6 \\
\hline Ensino médio completo & 4 & 6,3 \\
\hline Não lembra & 2 & 3,2 \\
\hline \multicolumn{3}{|l|}{ Estado civil } \\
\hline Solteiro & 13 & 20,7 \\
\hline Casado & 20 & 31,7 \\
\hline Estável & 3 & 4,8 \\
\hline Viúvo & 23 & 36,5 \\
\hline Divorciado/ separado & 4 & 6,3 \\
\hline \multicolumn{3}{|l|}{ Profissão/ ocupação } \\
\hline Aposentado/ pensionista & 38 & 60,3 \\
\hline Agricultor & 10 & 15,9 \\
\hline Do lar & 8 & 12,7 \\
\hline Outros & 7 & 11,1 \\
\hline \multicolumn{3}{|l|}{ Religião } \\
\hline Católico & 42 & 66,7 \\
\hline Evangélico & 19 & 30,1 \\
\hline Agnóstico & 2 & 3,2 \\
\hline Total & 63 & 100 \\
\hline
\end{tabular}

Os diagnósticos médicos encontrados com mais frequência nos pacientes foram: Acidente Vascular Encefálico (AVE) (39,0\%), Infecção do Trato Urinário
(17,5\%), Anemia (14,3\%), Infecção Respiratória Aguda $(12,7 \%)$ e Desnutrição (11,1\%). Quanto às doenças 
crônicas, 17,5\% dos pacientes possuíam Diabetes mellitus e 30,2\% Hipertensão Arterial Sistêmica.

A Escala de Independência em Atividades da Vida Diária (EIAVD) ou Escala de Katz permitiu identificar o nível de independência para a realização de atividades dentro das seis áreas de funcionamento: tomar banho, vestir-se, uso do vaso sanitário, transferência, continência e alimentação. Tal escala foi aplicada a todos os pacientes que participaram desse estudo e observou-se um maior nível de dependência nas áreas de funcionamento: uso do vaso sanitário $(96,8 \%)$, vestir-se e transferência, com frequência de 93,6\%, conforme mostra a Tabela 2 .

Tabela 2: Distribuição dos pacientes de acordo com a EIAVD. Vitória de Santo Antão, PE, 2012.

\begin{tabular}{lcccc}
\hline \multicolumn{1}{c}{ Área de funcionamento } & \multicolumn{2}{c}{ Independente } & \multicolumn{2}{c}{ Dependente } \\
Tomar banho & No & \% & No & 84,1 \\
Vestir-se & 10 & 15,9 & 53 & 93,6 \\
Uso do vaso sanitário & 4 & 6,3 & 59 & 96,8 \\
Transferência & 2 & 3,2 & 61 & 93,6 \\
Continência & 4 & 6,3 & 59 & 66,6 \\
Alimentação & 21 & 33,3 & 42 & 73,0 \\
\hline
\end{tabular}

Em relação ao Índice de Katz (IK), 60,3\% dos pacientes da amostra eram dependentes em todas as seis funções (IK-6), 27,5\% possuíam alguma dependência (IK-1,2,3,4 ou
5) e apenas $1,6 \%$ era independente em todas as seis funções (Tabela 3).

Tabela 3: Distribuição dos pacientes de acordo com a classificação da EIAVD ou Índice de Katz (IK). Vitória de Santo Antão, PE, 2012.

\begin{tabular}{lcc}
\hline \multicolumn{1}{c}{ Classificação } & $\mathbf{N}^{\mathbf{0}}$ & $\mathbf{\%}$ \\
\hline 0: independente em todas as 6 funções & 1 & 1,6 \\
1: independente em 5 funções e dependente em 1 função & 1 & 1,6 \\
2: independente em 4 funções e dependente em 2 função & 3 & 4,8 \\
3: independente em 3 funções e dependente em 3 função & 3 & 4,8 \\
4: independente em 2 funções e dependente em 4 função & 12,7 \\
5: independente em 1 funções e dependente em 5 função & 8 \\
6: dependente em todas as 6 funções & 14,3 \\
\hline
\end{tabular}

A partir do processo de raciocínio diagnóstico foram identificados44diagnósticos de enfermagem, com média de 17,1 diagnósticos por paciente. Estiveram presentes em todos os pacientes os diagnósticos de enfermagem: Deambulação prejudicada, Mobilidade física prejudicada, Risco de infecção e Risco de quedas. Outros diagnósticos com frequência elevada, acima do percentil 75, foram: Déficit no autocuidado para banho, Déficit no autocuidado para vestir-se, Déficit no autocuidado para higiene íntima, Capacidade de transferência prejudicada, Dentição prejudicada, Déficit no autocuidado para alimentação, Mobilidade no leito prejudicada e Eliminação urinária prejudicada. A Tabela 4 mostra os diagnósticos de enfermagem acima do percentil 50. 
Tabela 4: Distribuição dos diagnósticos de enfermagem acima do percentil 50 por pacientes. Vitória de Santo Antão, PE, 2012.

\begin{tabular}{lllc}
\hline \multicolumn{1}{c}{ Diagnóstico de enfermagem } & $\mathbf{N}^{\circ}$ & \% & IC (\%) \\
\hline Deambulação prejudicada & 63 & 100,0 & - \\
Mobilidade física prejudicada & 63 & 100,0 & - \\
Risco de infecção & 63 & 100,0 & - \\
Risco de queda & 63 & 100,0 & - \\
Déficit no autocuidado para banho & 61 & 96,8 & $92-100$ \\
Déficit no autocuidado para vestir-se & 60 & 95,2 & $89-100$ \\
Déficit no autocuidado para higiene íntima & 59 & 94,0 & $88-99$ \\
Capacidade de transferência prejudicada & 59 & 94,0 & $88-99$ \\
Dentição prejudicada & 58 & 92,1 & $85-98$ \\
Déficit no autocuidado para alimentação & 47 & 74,6 & $63-85$ \\
Mobilidade no leito prejudicada & 40 & 63,5 & $51-75$ \\
\hline Eliminação urinária prejudicada & 40 & 63,5 & $51-75$ \\
Interação social prejudicada & 38 & 60,3 & $48-72$ \\
Comunicação verbal prejudicada & 38 & 60,3 & $48-72$ \\
Nutrição desequilibrada: menos do que as necessidades corporais & 36 & 57,1 & $44-69$ \\
Estilo de vida sedentário & 36 & 57,1 & $44-69$ \\
Integridade da pele prejudicada & 28 & 44,4 & $27-51$ \\
Constipação & 25 & 39,7 & $27-51$ \\
Volume de líquido excessivo & 23 & 36,5 & $24-48$ \\
Deglutição prejudicada & 22 & 35,0 & $23-46$ \\
Conhecimento deficiente & 21 & 33,3 & $21-44$ \\
Intolerância a atividade & 21 & 33,3 & $21-44$ \\
\hline
\end{tabular}

\section{DISCUSSÃO}

Os resultados mostram um perfil de pacientes do sexo masculino (57,1\%), na faixa etária de 61 a 80 anos (42,9\%), analfabetos (44,4\%), viúvos (36,5\%), aposentados/pensionistas (60,3\%) e católicos (66,7\%). No que se refere ao diagnóstico médico encontrado nos pacientes, houve predominância de $\operatorname{AVE}(39,0 \%)$. Uma pesquisa realizada com o objetivo de traçar o perfil e os preditores para desfecho favorável de pacientes acometidos com AVE severo constatou que, dos 243 pacientes acometidos pela forma severa,53,5\% eram do sexo feminino e com média de idade de 69,6 anos $(\mathrm{DP}=16,8 \text { anos) })^{(16)}$.

Em um estudo realizado com 109 pacientes com AVE que apresentavam Mobilidade física prejudicada, foi observado que $52,3 \%$ pertenciam ao sexo masculino, $53,2 \%$ viviam com algum companheiro, $68,8 \%$ são aposentados ou pensionistas, com idade média de 62,1 $\operatorname{anos}^{(1)}$. Outro estudo realizado em unidade de clínica e cirúrgica, com pacientes com risco de úlcera por pressão, identificou que o principal motivo de internação dos pacientes foram as doenças cerebrovasculares $(22,4 \%)^{(17)}$.

A avaliação do nível de independência para realização de atividades da vida diária mostrou que a maioria dos pacientes $(60,3 \%)$ possuía dependência em todas as funções, e apenas $1,6 \%$ era independente em todas as funções. Em um estudo com o objetivo de identificar o nível de independência em AVDs em idosos institucionalizados em duas instituições de longa permanência, verificou-se que $23 \%$ dos idosos eram independentes nas seis funções, segundo o Índice de $\mathrm{Katz}^{(7)}$. Outra pesquisa, realizada com idosos residentes nas instituições da cidade de Taubaté-SP, verificou que $37 \%$ podiam ser considerados independentes ${ }^{(8)}$. Não foram encontrados estudos que fizessem relação com pacientes hospitalizados em clinica médica. Entretanto, a maioria dos pacientes que compuseram a amostra deste estudo é idosa (77,8\%).

Foram identificados 44 diagnósticos de enfermagem distintos. A correlação dos resultados obtidos na EIAVD com os diagnósticos de enfermagem encontrados, apontaram que $84,1 \%$ dos pacientes eram dependentes para tomar banho e $96,8 \%$ apresentaram o diagnóstico Déficit no autocuidado para banho, 93,6\% eram dependentes para vestir-se e 95,2\% apresentaram o diagnóstico Déficit no autocuidado para vestir-se, 74,6\% eram dependentes na função alimentação e apresentaram o diagnóstico Déficit no autocuidado para alimentação,93,6\% dos pacientes apresentavam dependência para a função transferência e $94 \%$ dos pacientes apresentaram o diagnóstico Capacidade de transferência prejudicada. 
Os diagnósticos de enfermagem Deambulação prejudicada e Mobilidade física prejudicada estiveram presentes em toda a amostra. De acordo com um estudo realizado com 194 pacientes em risco de desenvolverem úlcera por pressão, o diagnóstico Mobilidade física prejudicada esteve presente em $32 \%$ dos pacientes e estava relacionado com prejuízo neuromuscular e musculoesquelético, principalmente ${ }^{(17)}$. O diagnóstico de enfermagem Deambulação prejudicada, segundo um estudo realizado com 50 idosos submetidos a tratamento hemodialítico, foi identificado em $50 \%$ dos idosos ${ }^{(9)}$. Destaca-se que o fato destes diagnósticos estarem presentes em $100 \%$ da amostra condiz com a caracterização do estudo em pacientes com necessidade de locomoção afetada, por isso não foi possível encontrar resultados semelhantes.

O diagnóstico Risco de infecção foi identificado em toda a amostra do estudo. Sua elevada frequência justifica-se pelo fato da hospitalização expor o paciente a procedimentos invasivos e infecção cruzada, bem como a imobilidade favorecer a estase de fluidos corporais. Segundo levantamento dos diagnósticos de enfermagem para pacientes com risco de desenvolverem úlceras por pressão, o diagnóstico Risco de infecção foi o mais frequente em $55 \%$ dos pacientes e esteve relacionado com procedimentos invasivos em $97 \%$ dos $\operatorname{casos}^{(17)}$.

O Risco de quedas também foi identificado em $100 \%$ dos pacientes e representa um evento que pode comprometer a sua segurança. Em um estudo descritivo, realizado em um hospital universitário, verificou-se que as quedas do leito foram as mais frequentes (55\%), seguidas pelas quedas da própria altura (38,8\%). No que se refere às quedas do leito, as enfermarias de neurologia e clínica médica foram as mais frequentes com $22,7 \%$ e $20,4 \%$, respectivamente. Entretanto, as quedas da própria altura foram mais frequentes na clínica médica (38,7\%). O período noturno $(63,7 \%)$ obteve maior frequência ${ }^{(18)}$. É provável que as quedas ocorram com maior frequência no período noturno pela diminuição da visibilidade no ambiente, sonolência e necessidade de ir ao banheiro; fato que necessita de uma maior vigilância por parte da equipe de enfermagem neste período.

O Déficit no autocuidado para banho foi observado em $98,6 \%$ dos pacientes. De acordo com uma pesquisa em uma clínica médica de um Hospital Universitário, este diagnóstico esteve presente em $36 \%$ dos seus pacientes $^{(19)}$. O déficit no autocuidado para vestir-se e o déficit no autocuidado para higiene íntima foram identificados em $95,2 \%$ e $94,0 \%$ dos pacientes, respectivamente. Uma pesquisa desenvolvida em uma clínica ortopédica, onde os pacientes também tinham necessidade de locomoção afetada, mostrou que $45 \%$ apresentaram déficit no autocuidado para vestir-se e $25 \%$ déficit no autocuidado para higiene íntima ${ }^{(20)}$. 0 déficit no autocuidado reflete a necessidade da presença de um acompanhante diariamente e o papel da Enfermagem também é muito importante para a prestação de orientações aos acompanhantes, além do cuidado direcionado para o próprio paciente.

O diagnóstico de enfermagem Capacidade de transferência prejudicada foi identificado em $94 \%$ da amostra e com maior frequência esteve relacionado à força muscular insuficiente e prejuízo neuromuscular. Um estudo em pacientes com sequelas de AVE constatou que nos pacientes que tinham um cuidador, a probabilidade do surgimento desse diagnóstico aumentou aproximadamente três vezes, pelo fato do cuidador desempenhar um papel de superproteção e realizar as atividades que deveriam ser realizadas pelo paciente ${ }^{(21)}$.É importante que o cuidador estimule e permita que o paciente realize o maior número possível de atividades da vida diária, de acordo com suas limitações, mas no intuito de torná-lo o mais independente possível.

A Dentição prejudicada caracterizou-se por presença de cáries, dentição incompleta e ausência de dentes ocasionada por higiene oral ineficaz, falta de acesso a cuidados profissionais, conhecimento deficiente acerca da saúde bucal, barreiras ao autocuidado e econômicas, presente em $92,1 \%$ dos pacientes. De acordo com um estudo em idosos dependentes, esse diagnóstico foi identificado em $100 \%$ da amostra ${ }^{(22)}$. Esse resultado remete ao fato da maior parte dos pacientes serem idosos e, comumente, apresentarem problemas relacionados à dentição. Estudo aponta que, com o avanço da idade, aumenta-se o número de dentes ausentes e isto não decorre do processo de envelhecimento natural, pois os dentes, com os seus devidos cuidados, podem permanecer intactos durante toda a vida ${ }^{(23)}$.

O Déficit no autocuidado para alimentação esteve relacionado, principalmente, a prejuízo cognitivo e neuromuscular, na maioria das vezes, associado à sequela de AVE. Um estudo identificou que a função física e a 
nutrição são os domínios mais frequentemente associados à mortalidade a curto prazo de idosos institucionalizados e hospitalizados(24). Como consequência, os pacientes que apresentam esse diagnóstico de enfermagem podem desenvolver problemas nutricionais. É importante que sejam realizadas medidas preventivas e que amenizem o déficit no autocuidado para a alimentação.

O diagnóstico de enfermagem Mobilidade no leito prejudicada foi identificado em $63,5 \%$ dos pacientes e, de acordo com um estudo com 13 pacientes com risco de desenvolverem úlcera por pressão, identificou-se que $80 \%$ dos pacientes possuía esse diagnóstico(25). Na maioria das vezes, quando o paciente apresenta esse diagnóstico e não são realizados os cuidados necessários, ocorre o aparecimento das úlceras por pressão, as quais aumentam o tempo de permanência hospitalar e podem acarretar outras complicações de saúde.

\section{CONSIDERAÇÕES FINAIS}

No estudo foram avaliados 63 pacientes com necessidade de locomoção afetada internados em uma unidade de clínica médica. No que se refere à independência em atividades da vida diária, a maioria dos pacientes possuía dependência em todas as funções avaliadas (tomar banho, vestir-se, uso do vaso sanitário, transferência, continência, alimentação). Foram identificados 44 diagnósticos de enfermagem distintos. Estiveram presentes em todos os pacientes os

\section{REFERÊNCIAS}

1. Costa AGS, Oliveira ARS, Alves FEC, Chaves DBR, Moreira RP, Araújo TL. Diagnóstico de enfermagem: mobilidade física prejudicada em pacientes acometidos por acidente vascular encefálico. Rev Esc Enferm USP [Internet]. 2010 [acesso em: 20 dez 2013];44(3):753-8. Disponível em:

http://dx.doi.org/10.1590/S0080-62342010000300029.

2. França DC, Apolinário AQ, Velloso M, Parreira VF. Reabilitação pulmonar na unidade de terapia intensiva: revisão de literatura. Fisioter. Pesqui. [Internet]. 2010 [acesso em: $20 \mathrm{dez}$ 2013];17(1):81-7. Disponível em:

http://dx.doi.org/10.1590/S1809-29502010000100015. 3. Morais EM, Marino MCA, Santos RR. Principais síndromes geriátricas. Rev Med Minas Gerais[Internet]. 2010 [acesso em: 20 dez 2013];20(1):54-66. Disponível em: http://rmmg.medicina.ufmg.br/index.php/rmmg/article/viewArt icle/208.

4. Salomão GSM, Azevedo RCS. Produção bibliográfica sobre o processo de enfermagem. Acta paul. enferm. [Internet]. 2009 [acesso em: $20 \mathrm{dez}$ 2013];22(5):691-5. Disponível em: http://dx.doi.org/10.1590/S0103-21002009000500015. diagnósticos de enfermagem Deambulação prejudicada, Mobilidade física prejudicada, Risco de infecção e Risco de quedas.

O conjunto de diagnósticos identificados reforça a importância do processo de enfermagem na abordagem aos pacientes, por proporcionar a individualização do cuidado e é relevante para orientar a assistência de enfermagem com base nas necessidades afetadas, de modo a garantir o bem estar físico, mental e social. Portanto, é importante que o enfermeiro esteja apto a identificar qualquer alteração que possa acometer indivíduos com necessidade de locomoção afetada e intervir mesmo antes de seu surgimento, por meio de intervenções preventivas.

Esse estudo limitou-se a pacientes com necessidade de locomoção afetada em uma unidade de clinica médica. Assim, recomenda-se a realização de outros estudos com pacientes com necessidade de locomoção afetada internados em outras realidades para que seja traçado um perfil destes pacientes e, assim, melhor direcionar o cuidado a eles prestado. Estudos com a finalidade de traçar perfil de diagnósticos de enfermagem em uma dada população apresentam como fragilidade o fato dos instrumentos para coleta de dados serem limitados, pois não são capazes de identificar todos os diagnósticos de enfermagem da NANDA-I. Para minimizar este viés, recomenda-se a utilização de instrumentos previamente validados, conforme utilizados neste estudo.

5. Resolução COFEN 358/2009 (BR). Dispõe sobre a Sistematização da Assistência de Enfermagem e a implementação do Processo de Enfermagem em ambientes, públicos ou privados, em que ocorre o cuidado profissional de Enfermagem, e dá outras providências. Conselho Federal de Enfermagem [Internet]. 15 de outubro de 2009 [acesso em: 20 dez 2013]. Disponível em:

http://novo.portalcofen.gov.br/resoluo-cofen-

3582009 4384.html.

6. Brito MAGM, Bachion MM, Souza JT. Diagnósticos de enfermagem de maior ocorrência em pessoas com lesão medular no contexto do atendimento ambulatorial mediante abordagem baseada no modelo de Orem. Rev. Eletr. Enf. [Internet]. 2008 [acesso em: $20 \mathrm{dez} 2013$ ];10(1):13-28. Disponivel em:

http://www.fen.ufg.br/revista/v10/n1/v10n1a02.htm.

7. Lisboa CR, Chianca TCM. Perfil epidemiológico, clínico e de independência funcional de uma população idosa institucionalizada. Rev Bras Enferm [Internet]. 2012 [acesso em: 20 dez 2013];65(3):482-8. Disponível em: http://dx.doi.org/10.1590/S0034-71672012000300013. 
8. Araújo MOPH, Ceolim MF. Avaliação do grau de independência de idosos residentes em instituições de longa permanência. Rev Esc Enferm USP [Internet]. 2007 [acesso em: 20 dez 2013];41(3):378-85. Disponível em: http://dx.doi.org/10.1590/S0080-62342007000300006. 9. Fernandes MGM, Pereira MA, Bastos RAA, Santos KFO. Diagnósticos de enfermagem do domínio atividade/repouso evidenciados por idosos em tratamento hemodialítico. Rev Rene [Internet]. 2012 [acesso em: 20 dez 2013];13(4):929-37. Disponível em:

http://www.revistarene.ufc.br/revista/index.php/revista/article/ view/77.

10. Marin MJS, Cecílio LCO, Rodrigues LCR, Ricci FA, Druzian S. Diagnósticos de enfermagem de idosas carentes de um programa de saúde da família (PSF). Esc. Anna Nery [Internet]. 2008 [acesso em: 20 dez 2013];12(2):278-84. Disponível em: http://dx.doi.org/10.1590/S1414-81452008000200012. 11. Benedet SA, Brasil N. A sistematização da assistência de enfermagem e as necessidades de cuidados de pacientes internados em terapia intensiva. Revista Gestão \& Saúde [Internet]. 2012 [acesso em: 20 dez 2013];3(2):800-15. Disponivel em:

http://www.gestaoesaude.unb.br/index.php/gestaoesaude/arti cle/view/169.

12. Virgínio NA, Nóbrega MML. Validação de instrumento de coleta de dados de enfermagem para clientes adultos hospitalizados. Rev Bras Enferm [Internet]. 2004 [acesso em: 20 dez 2013];57(1):53-6. Disponível em:

http://dx.doi.org/10.1590/S0034-71672004000100011. 13. Lino VTS, Pereira SRM, Camacho LAB, Ribeiro Filho ST, Buksman S. Adaptação transcultural da Escala de Independência em Atividades da Vida Diária (Escala de Katz). Cad Saude Publica [Internet]. 2008 [acesso em: $20 \mathrm{dez} 2013$ ];24(1):103-12. Disponivel em: http://dx.doi.org/10.1590/S0102311X2008000100010.

14. Gordon M. Nursing diagnosis: process and application. $3^{\text {a }}$ ed. St Louis: Mosby; 1994.

15. NANDA International. Diagnósticos de enfermagem da NANDA. Definições e Classificação 2012-2014. Porto Alegre: Artmed; 2013. $606 \mathrm{p}$.

16. Bill O, Zufferey P, Faouzi M, Michel P.Severe stroke: patient profile and predictors of favorable outcome. J Thromb Haemost [Internet]. 2013 [acesso em: 20 dez 2013];11(1):92-9. Disponível em: http://dx.doi.org/10.1111/jth.12066.

17. Lucena AF, Santos CT, Pereira AGS, Almeida MA, Dias VLM, Friedrich MA. Clinical profile and nursing diagnosis of patients at risk of pressure ulcers. Rev Lat Am Enfermagem [Internet]. 2011 [acesso em: 20 dez 2013];19(3):523-30. Disponível em: http://dx.doi.org/10.1590/S0104-11692011000300011. 18. Paiva MCMS, Paiva SAR, Berti HW, Campana AO. Caracterização das quedas de pacientes segundo notificação em boletins de eventos adversos. Rev Esc Enferm USP [Internet]. 2010 [acesso em: $20 \mathrm{dez} 2013$ ];44(1):134-8. Disponível em: http://dx.doi.org/10.1590/S0080-62342010000100019. 19. Lima AFC, Fugulin FMT, Castilho V, Nomura FH, Gaidzinski RR. Contribuição da documentação eletrônica de enfermagem para aferição dos custos dos cuidados de higiene corporal. J. Health Inform. [Internet]. 2012 [acesso em: $20 \mathrm{dez}$ 2013];4(Número Especial - SIIENF 2012):108-13. Disponível em: http://www.jhi-sbis.saude.ws/ojs-jhi/index.php/jhisbis/article/view/239.

20. Silva FS, Fernandes MV, Volpato MP. Diagnósticos de enfermagem em pacientes internados pela clínica ortopédica em unidade médico-cirúrgica. Rev Gaucha Enferm [Internet]. 2008 [acesso em: 20 dez 2013];29(4):565-72. Disponível em: http://seer.ufrgs.br/RevistaGauchadeEnfermagem/article/view/ $\underline{3826}$.
21. Moreira RP, Araujo TL, Cavalcante TF, Oliveira ARS, Holanda GF, Morais HCC et al. Cuidador de cliente com acidente vascular encefálico: associação com diagnósticos de enfermagem. Rev. Eletr. Enf. [Internet] 2010 [acesso em: $20 \mathrm{dez}$ 2013];12(3):42530. Disponível em: http://dx.doi.org/10.5216/ree.v12i3.6391. 22. Freitas MC, Pereira RF, Guedes MVC. Diagnósticos de enfermagem em idosos dependentes residentes em uma instituição de longa permanência em Fortaleza-CE. Ciênc. cuid. saúde [Internet] 2010 [acesso em: $20 \mathrm{dez}$ 2013];9(3):51826.Disponivel em: http://dx.doi.org/10.4025/cienccuidsaude.v9i3.9544. 23. Guedes HM, Nakatani AYK, Santana RF, Bachion MM. Identificação de diagnósticos de enfermagem do domínio segurança/proteção em idosos admitidos no sistema hospitalar. Rev. Eletr. Enf. [Internet]. 2009 [acesso em: $20 \mathrm{dez}$ 2013];11(2):249-56. Disponível em: http://www.fen.ufg.br/revista/v11/n2/v11n2a03.htm. 24. Thomas JM, Cooney LM Jr, Fried TR. Systematic review: Health-related characteristics of elderly hospitalized adults and nursing home residents associated with short-term mortality. J Am Geriatr Soc [Internet]. 2013 [acesso em: $20 \mathrm{dez}$ 2013];61(6):902-11. Disponível em: http://dx.doi.org/10.1111/jgs.12273. 25. Araújo TM, Araújo MFM, Caetano JA, Galvão MTG, Damasceno MMC. Diagnósticos de enfermagem para pacientes em risco de desenvolver úlcera por pressão. Rev Bras Enferm [Internet]. 2011 [acesso em: $20 \mathrm{dez} 2013$ ];64(4):671-6. Disponível em: http://dx.doi.org/10.1590/S0034$\underline{71672011000400007}$.

Artigo recebido em 15/05/2013.

Aprovado para publicação em 16/09/2013.

Artigo publicado em 31/12/2013. 